University of Wollongong

Research Online

Faculty of Engineering and Information

Faculty of Engineering and Information

Sciences - Papers: Part A

Sciences

2014

Modeling and inverse feedforward control for conducting polymer actuators with hysteresis

Xiangjiang Wang

University of Wollongong, xwang@uow.edu.au

Gursel Alici

University of Wollongong, gursel@uow.edu.au

Xiaobo Tan

Michigan State University

Follow this and additional works at: https://ro.uow.edu.au/eispapers

Part of the Engineering Commons, and the Science and Technology Studies Commons

Research Online is the open access institutional repository for the University of Wollongong. For further information contact the UOW Library: research-pubs@uow.edu.au 


\title{
Modeling and inverse feedforward control for conducting polymer actuators with hysteresis
}

\begin{abstract}
Conducting polymer actuators are biocompatible with a small footprint, and operate in air or liquid media under low actuation voltages. This makes them excellent actuators for macro- and micro-manipulation devices, however, their positioning ability or accuracy is adversely affected by their hysteresis nonlinearity under open-loop control strategies. In this paper, we establish a hysteresis model for conducting polymer actuators, based on a rate-independent hysteresis model known as the Duhem model. The hysteresis model is experimentally identified and integrated with the linear dynamics of the actuator. This combined model is inverted to control the displacement of the tri-layer actuators considered in this study, without using any external feedback. The inversion requires an inverse hysteresis model which was experimentally identified using an inverse neural network model. Experimental results show that the position tracking errors are reduced by more than $50 \%$ when the hysteresis inverse model is incorporated into an inversion-based feedforward controller, indicating the potential of the proposed method in enabling wider use of such smart actuators.
\end{abstract}

\section{Keywords}

control, feedforward, inverse, hysteresis, modeling, actuators, polymer, conducting

Disciplines

Engineering | Science and Technology Studies

\section{Publication Details}

Wang, X., Alici, G. \& Tan, X. (2014). Modeling and inverse feedforward control for conducting polymer actuators with hysteresis. Smart Materials and Structures, 23 (2), 025015-025023. 


\title{
Modeling and Inverse Feedforward Control for Conducting Polymer
}

\author{
Actuators with Hysteresis \\ Xiangjiang Wang ${ }^{\mathrm{a}, \mathrm{b}}$, Gursel Alici ${ }^{\mathrm{b}}$, Xiaobo $\operatorname{Tan}^{\mathrm{c}}$ \\ ${ }^{\mathrm{a}}$ School of Mechanical Engineering, University of South China, Hengyang 421001, China \\ ${ }^{\mathrm{b}}$ School of Mechanical, Materials and Mechatronic Engineering, University of Wollongong, 2522 NSW, Australia \\ ${ }^{c}$ Department of Electrical \& Computer Engineering, Michigan State University, East Lansing, MI 48824, USA
}

Corresponding Author: G. Alici, Email: gursel@uow.edu.au

\begin{abstract}
Conducting polymer actuators are biocompatible with a small foot-print, and operate in air or liquid media under low actuation voltages. This makes them excellent actuators for macro- and micro-manipulation devices, however, their positioning ability or accuracy is adversely affected by their hysteresis non-linearity under open-loop control strategies. In this paper, we establish a hysteresis model for conducting polymer actuators, based on a rate-independent hysteresis model known as the Duhem model. The hysteresis model is experimentally identified and integrated with the linear dynamics of the actuator. This combined model is inverted to control the displacement of the tri-layer actuators considered in this study, without using any external feedback. The inversion requires an inverse hysteresis model which was experimentally identified using an inverse neural network model. Experimental results show that the position tracking errors are reduced by more than $50 \%$ when the hysteresis inverse model is incorporated into an inversion-based feedforward controller, indicating the potential of the proposed method in enabling wider use of such smart actuators.
\end{abstract}

Keywords-Conducting polymer actuators; hysteresis model; Duhem model; system identification; inversion-based feedforward control.

\section{Introduction}

Conducting polymer actuators have received significant attention [1]-[4] in the past decade due to their low operating voltage, inherent biocompatibility, high force output to weight ratio, noiseless operation with a small footprint, and insensitivity to magnetic fields. These actuators could be engineered as bending actuators like a cantilever beam, which are suitable for activating micro-pumps, micro-switches, micro-grippers and micro-cantilevers, to move, position or hold micro devices and objects, and manipulate biological samples. Mathematical models have previously been developed to understand their quasi-static and dynamic behaviors and establish feedback control strategies [5]-[7]. Feedback control and open-loop control (using an inverse model of the actuator) techniques can be employed to control the actuator's bending displacement or bending angle [8]-[10]. In particular, open-loop control techniques are desirable for such smart actuators since it is not practical to use an external sensor for feedback in most of the applications envisioned. With this in mind, it is crucial to model non-linear effects such as hysteresis to enhance the accuracy of the actuator model and hence increase the positioning ability of these actuators without using an external sensor.

When conducting polymer actuators are influenced by their hysteresis non-linearity, the whole system usually exhibits undesirable inaccuracies. The hysteresis phenomenon occurs in 
almost all smart material-based actuators [11]-[15]. Researchers have proposed various methods for hysteresis modeling, however, hysteresis modeling has not been reported for tri-layer conducting polymer actuators. The Preisach model and various other hysteresis models are widely used [16]-[18], but these operators are typically difficult to identify and invert [19]-[20]. On the other hand, the Duhem hysteresis model is simpler to construct and experimentally identify $[21,28]$. Over the years, various control techniques have been developed to mitigate the effects of hysteresis. Much of this renewed interest is a direct consequence of the importance of smart materials in numerous current applications. The interest in studying dynamic systems with actuator hysteresis is also motivated by the fact that they are non-linear systems with non-smooth non-linearities for which traditional control methods are insufficient and thus require the development of alternative approaches.

In this paper, we develop a method for building a hysteresis model for tri-layer conducting polymer actuators. The model consists of two sub-systems, a rate-independent Duhem hysteresis model and a linear dynamic model. The efficacy of the model is demonstrated with experimental results obtained from the actuators controlled through an inversion-based feedforward controller. It must be noted the success of the feedforward control depends on the accuracy of the mathematical model describing the dynamic behaviour of the actuators. As reported before [5,10,23,24,29], it is virtually impossible to have a mathematical model accounting for a wide range of frequencies and the variations in the electrical, chemical and mechanical parameters of these tri-layer actuators including their nonlinear behaviour such as creep and hysteresis. We have previously reported on a hysteresis model to be used in the feedforward control of these actuators [28], and other smart actuators whose dynamic behaviour and hysteretic behaviour can similarly be modelled and identified, and subsequently it can be used in a feedforward controller in order to control the displacement output of these actuators. This paper incorporates this hysteresis model into the linear model of the actuator to establish a more accurate mathematical model, for the sensorless position control of these actuators.

\section{Experimental Setup}

Figure 1 shows the structure of the bending-type trilayer polymer actuators considered in this study. It consists of two outer polypyrrole (PPy) layers that are the active components (serving as electrodes), an inner porous separator of PVDF that holds the liquid electrolyte, and two sputter-coated gold layers with negligible thickness for electrochemically growing the polymer layers on the PVDF layer. The bending actuator with the desired length and width is cut from the bulk sheet. This composite structure exhibits a simple bending motion like a bi-layer cantilever. When a potential difference or current is passed between the polymer (PPy) electrodes via the clamp, the polymer actuator bends and outputs a mechanical motion at its tip, due to an electrochemical reaction. We have presented the procedure for the synthesis of the actuators in our previous study [22]. Such bending actuators are suitable for moving, positioning or holding micro devices, objects and biological samples. In particular, due to their compliance, they can be used as a cell-tapper to mechanically stimulate a single cell $[25,26]$. 


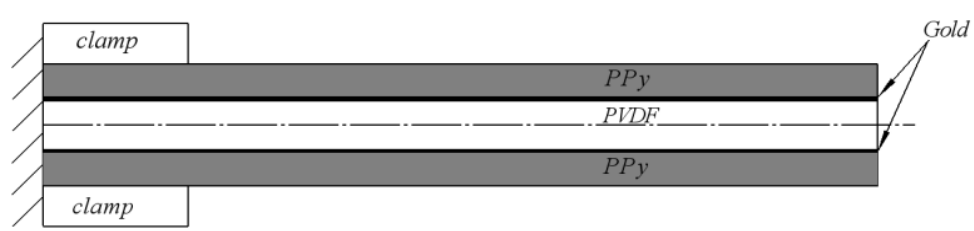

Figure 1. Geometry of the conducting polymer actuator.

Figure 2 shows the experimental set-up for model identification and control. The input voltage applied to the actuator is first generated by the software and then transmitted to an NI USB-6251 DAQ board and to an eDAQ potentiostat (eDAQ, model EA161) operating in the two-electrode mode. A non-contact laser sensor (Micro-epsilon, model NCDT-1700-10) is used to measure the tip displacement of the actuator. The data is sent to the computer via a data logger (e-Corder, model ED821). The control system is built in MATLAB which is connected to the experimental hardware through object linking.

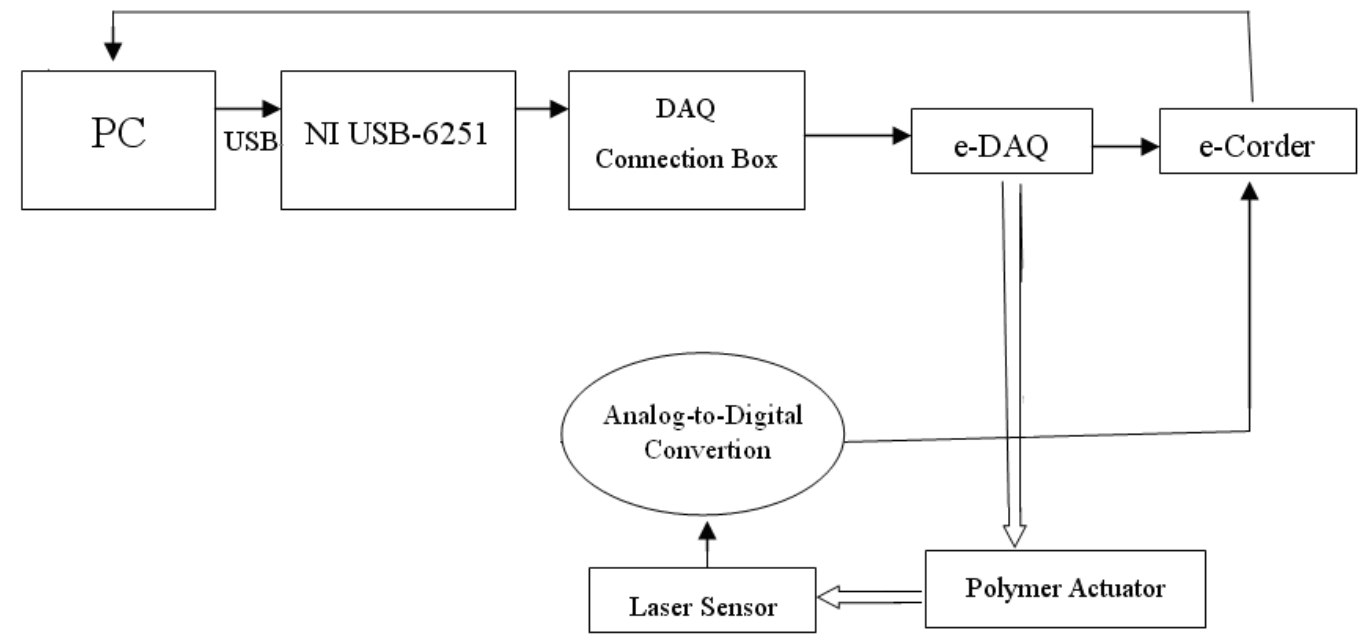

Figure 2. Schematic representation of the experimental set-up.

It is usually difficult to build accurate analytical or physical models due to non-linearities and multi-physics (electro-chemo-mechanical) phenomena associated with the actuator. It is feasible, however, to build a system model empirically that is adequate for control system design, analysis, and implementation.

\section{Actuator Model}

To identify the mathematical model of a tri-layer PPy actuator, several sinusoidal voltages were applied to a $15 \mathrm{~mm}$-long, $5 \mathrm{~mm}$-wide, $0.17 \mathrm{~mm}$-thick actuator. The input voltage signal of $u(t)=\sin (\omega t)$, with a range of frequencies, $\omega(\mathrm{rad} / \mathrm{sec})=0.5 \pi, 1.0 \pi, 2.0 \pi$, was chosen. The relationship between the input voltage and output displacement $y(t)$ can be used to obtain a transfer function model of the actuator.

\subsection{Identifying Discrete-Time Transfer Function}

By ignoring non-linearities [10], the actuator can be first modeled as a linear system which can be represented by a discrete-time transfer function $G_{1}(z)$ shown in Figure 3. Considering the relationship between the input $u(k)$ and output $y(k)$ to be a "black box", one can experimentally identify the transfer function in a straightforward manner. 


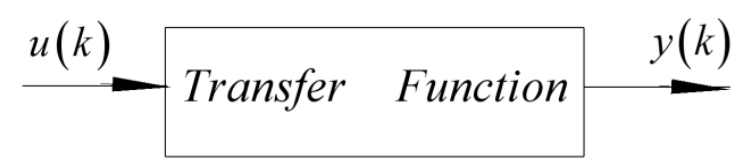

Figure 3. Transfer function representation of the actuator.

We can employ offline identification techniques to identify the discrete-time transfer function. Here a least-square estimation method is used. As we reported previously [8, 10, 23, 24], although the dynamics of conducting polymer actuators is of high order, it can be approximated with a second-order system;

$$
G_{1}(z)=\frac{b_{10} z+b_{11}}{z^{2}+a_{11} z+a_{12}}
$$

Eq. (1) implies

$$
A(z) y(t)=B(z) u(t)
$$

With $A(z)=1+a_{11} z^{-1}+a_{12} z^{-2}$

$$
B(z)=b_{10} z^{-1}+b_{11} z^{-2}
$$

The term $z^{-1}$ is the unit delay operator, and $z^{-1} y(t)=y(t-1)$

We employ the least-square method to identify the parameters of the actuator system. The parameter vector $\theta$ is defined as

$$
\theta^{T}=\left[a_{11}, a_{12} ; b_{10}, b_{11}\right]
$$

The regression vector is described as

$$
\varphi^{T}(t)=[-y(t-1),-y(t-2) ; u(t-1), u(t-2)]
$$

Eq. (1) can be written as

$$
y_{t}=H_{t} \theta
$$

with

$$
y_{t}=\left[\begin{array}{c}
y(1) \\
y(2) \\
\vdots \\
y(t)
\end{array}\right], \quad H_{t}=\left[\begin{array}{c}
\varphi^{T}(1) \\
\varphi^{T}(2) \\
\vdots \\
\varphi^{T}(t)
\end{array}\right]
$$

According to the least squares criterion, the estimated value of parameter vector $\theta$ is

$$
\hat{\theta}=\left(H_{t}^{T} H_{t}\right)^{-1} H_{t}^{T} y_{t}
$$

This linear model can be used to simulate the bending displacement under a voltage input. The model identification errors are shown in Figure 4. The error $e(t)$ is the error 
between the real output $y(t)$ and the predicted output $y_{1}(t)$. We see that the output predicted through the linear transfer function model deviates significantly from the experimental output. One reason for modeling errors is the unmodelled non-linearities such as the hysteresis, which has generally been neglected in previously reported models.

The dynamics of conducting polymer actuators can accurately be represented by a high model (at least $4^{\text {th }}$ order, [10], [24]) for an acceptable bandwidth $(\sim 38 \mathrm{~Hz})$. The numerical values of the parameters for the second-order model in Eq.1 are experimentally identified and provided in Table 1. It must also be noted that the magnitude of the input displacement is kept low such that the actuator can show a linear dynamic behavior $[8,10]$, which can be identified by using linear system modeling and identification techniques.

Table 1. The numerical values of the model parameters of Eq.1

\begin{tabular}{|c|c|c|c|}
\hline$b_{10}$ & $b_{11}$ & $a_{11}$ & $a_{12}$ \\
\hline 0.0352 & -0.0318 & -1.0831 & 0.0859 \\
\hline
\end{tabular}

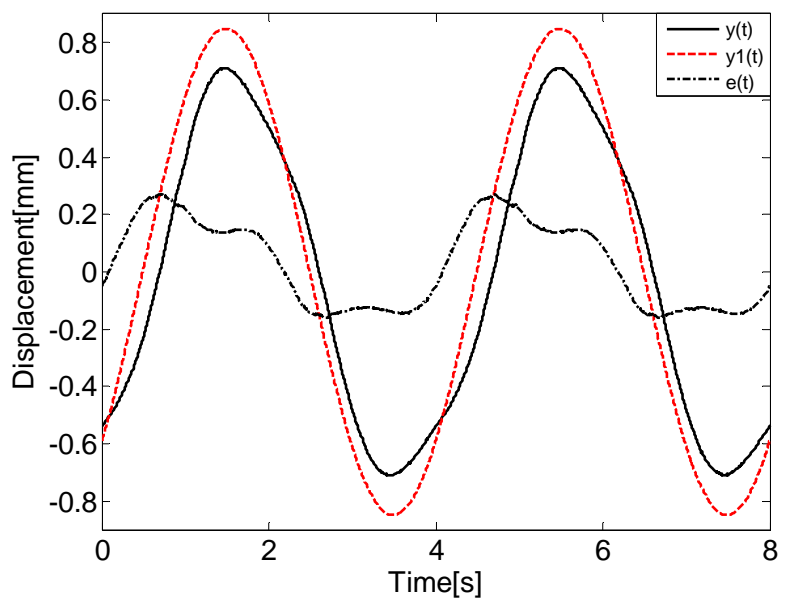

(a) Input $u(t)=\sin (0.5 \pi t)$

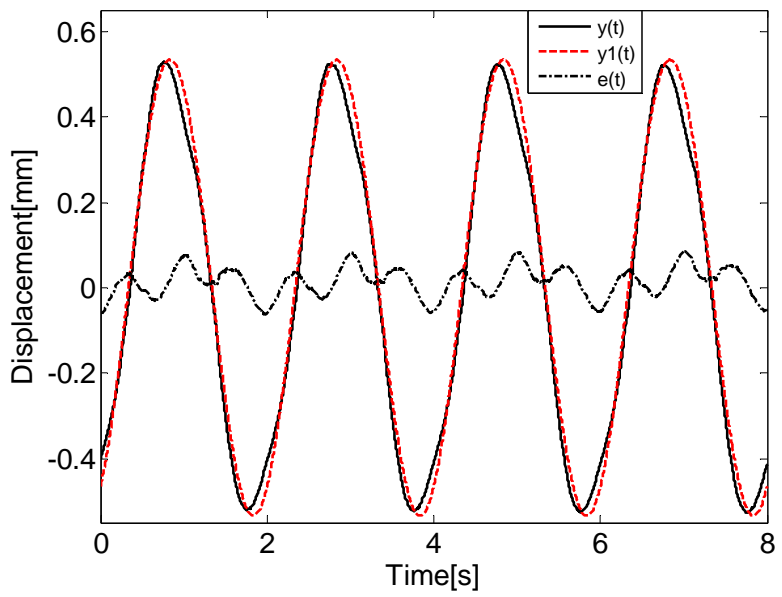


(b) Input $u(t)=\sin (\pi t)$

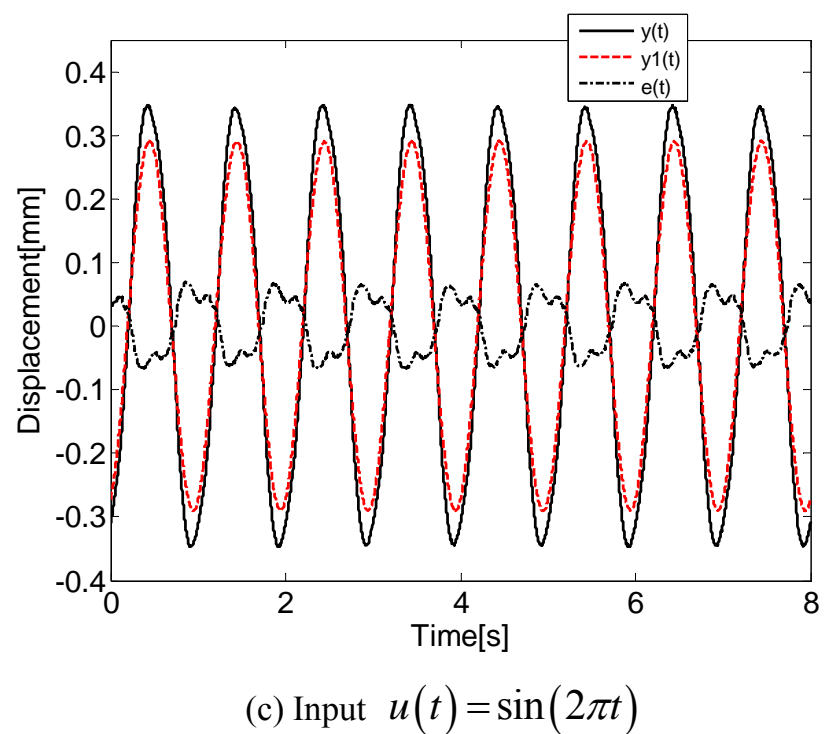

Figure 4. Comparison of the predicted actuator displacement output with the experimental displacement output under sinusoidal inputs with different frequencies.

\subsection{Hysteresis model incorporated into actuator model}

To improve the accuracy of the actuator model, the influence of the hysteresis non-linearity needs to be modeled. We consider a transfer function $G_{2}(z)$ connected in cascade to a hysteresis model $H(t)$, as shown in Figure 5.

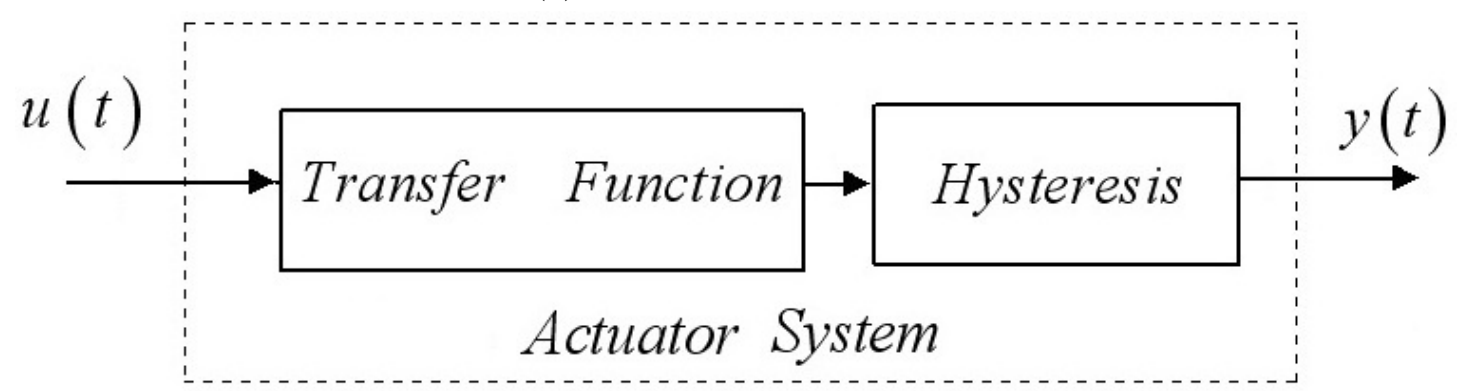

Figure 5. The block diagram of the actuator containing hysteresis non-linearities.

The time shift between the fundamental frequency component of the displacement output $y(t)$ and input voltage $u(t)$ is $\Delta t$, which is determined by comparing the peaks of experimental displacement signal and the input voltage. To eliminate the effect of the phase shift introduced by the transfer function, the input signal needs to be given a backward phase shift [27]; in this case, the phase shift will be of $\varphi(\omega)=\omega \Delta t$, which results in the input for the hysteresis model

$$
v(t)=\sin (\omega t-\omega \Delta t)
$$

Note that, with (7), one ensures the output of the hysteresis reaches maximum (minimum, resp.) when the input reaches maximum (minimum, resp.), which is a necessary condition for a rate-independent, monotonic hysteresis operator. Also, to eliminate the frequency-dependent amplitude property of the linear part, the output data is normalized as 
follows;

$$
w(t)=\frac{y(t)}{k}
$$

where $k=\max (|y(t)|)$. With (8), we ensure that the output $w(t)$ of the hysteresis has the same range (namely, $[-1,1]$, in this case) when its input is of different frequencies but of same amplitude, which is a necessary condition for the rate-independent hysteresis model.

After adjusting the input and output signals, the hysteresis curves are obtained as shown in Figure 6, where $v(t)$ is the input signal and $w(t)$ is the output signal. The hysteresis curves are frequency or rate-independent as they show very similar hysteresis behaviour under different input frequencies. The discrepancy between the hysteresis loops in Figure 6 is negligibly small.

The hysteresis phenomenon occurs in almost all the smart material-based actuators, such as piezoceramics, MR and magnetostrictive materials. With this nonlinearity, the whole system usually exhibits undesirable inaccuracies, oscillations or even instability. Over many years, various control techniques had been developed to mitigate the effects of hysteresis and recently these have again attracted significant attention from both industry and academic research. Much of this renewed interest is a direct consequence of the importance of hysteresis in numerous applications.

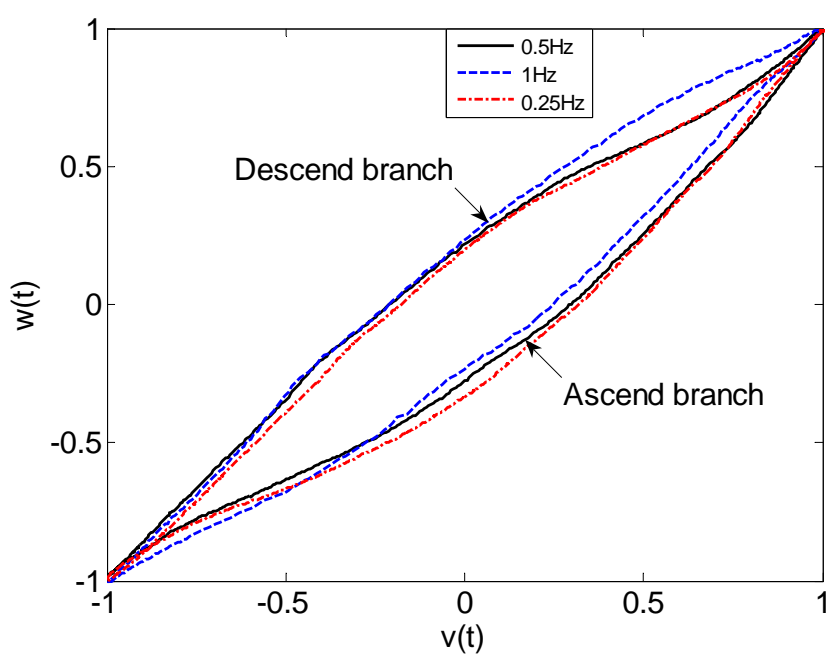

Figure 6. Hysteresis loops of the actuator under different input frequencies.

\subsubsection{Modeling the hysteresis}

We employ the Duhem hysteresis model to mathematically represent the hysteresis loops in Figure 6. The generalized Duhem model is given by [21,28]:

$$
\frac{d w}{d t}=\alpha\left|\frac{d v}{d t}\right|[f(v)-w]+\frac{d v}{d t} g(v)
$$

where $f(v)$ and $g(v)$ are the characteristic functions of the hysteresis mode, $v(t)$ is the input signal, and $w(t)$ is the output signal. Eq. (9) can also be written as

$$
\frac{d w_{A}}{d v}=\alpha\left[f(v)-w_{A}\right]+g(v), \quad \dot{v}>0
$$




$$
\frac{d w_{D}}{d v}=-\alpha\left[f(v)-w_{D}\right]+g(v), \quad \dot{v}<0
$$

where, $w_{A}(t)$ and $w_{D}(t)$ are the mathematical functions describing the ascending and descending branches, respectively. It follows that the characteristic functions are obtained as

$$
\begin{aligned}
& f(v)=\frac{1}{2}\left(w_{A}+w_{D}\right)+\frac{1}{2 \alpha}\left(\frac{d w_{A}}{d v}-\frac{d w_{D}}{d v}\right) \\
& g(v)=\frac{1}{2}\left(\frac{d w_{A}}{d v}+\frac{d w_{D}}{d v}\right)+\frac{1}{2} \alpha\left(w_{A}-w_{D}\right)
\end{aligned}
$$

Based on Eq.(12) and Eq.(13), the functions $f(v)$ and $g(v)$ in the Duhem model can be identified from a set of experiment data. $w_{A}(t)$ and $w_{D}(t)$ as well as their derivatives are needed to identify $f(v)$ and $g(v)$.

In equations (9)-(13), $\alpha$, which is a positive number, can be regarded as a weighting factor. When identifying $f(v)$ and $g(v)$, a larger error arises in the evaluation of the derivative curves than in the ascending or descending branches of the hysteresis loop. With this in mind, a large value of $\alpha=80$ is selected to minimize the entire identification error. A neural network is employed to improve the accuracy of the identification functions $f(v)$ and $g(v)$, and to identify the ascending branch $w_{A}(t)$ and descending branch $w_{D}(t)$ as shown in Figure 6.

The neural network identification method is used to build $f(v)$ and $g(v)$. The hysteresis curves shown in Figure 7 are the simulated results calculated from the Duhem hysteresis model of Eq. (9), which is close to the experimental results shown in Figure 6 $(0.5 \mathrm{~Hz})$. It leads to the conclusion that the hysteresis modeling method is effective in representing the hysteresis of the conducting polymer actuators considered in this study.

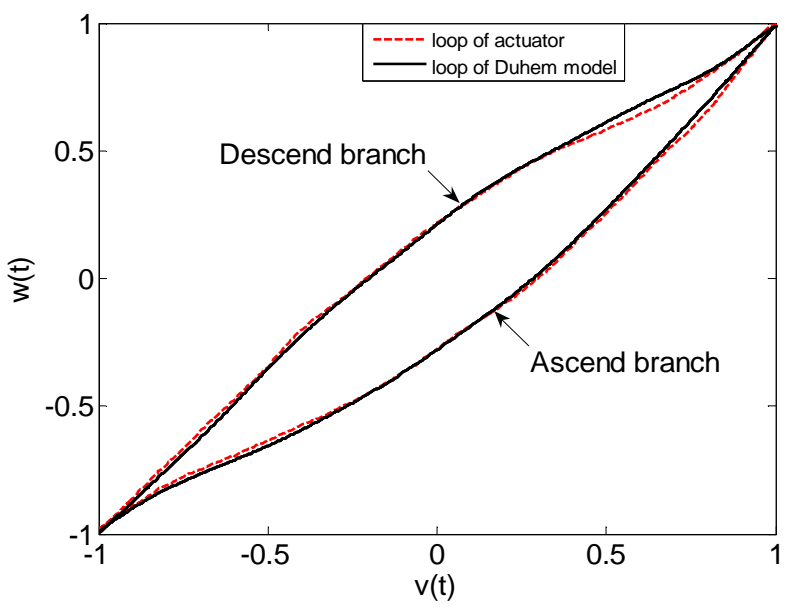

Figure 7. Hysteresis loop of Duhem model

\subsubsection{Building the transfer function of the linear part for non-linear system}

In order to compensate for the hysteresis effect in the actuator, represented by the block diagram in Figure 5, the hysteresis inverse model must be established and experimentally identified. The Duhem hysteresis inverse model can be expressed as: 


$$
\frac{d q(t)}{d t}=\alpha\left|\frac{d w}{d t}\right|[F(w)-q(t)]+\frac{d w}{d t} G(w)
$$

where $F(w)$ and $G(w)$ are the characteristic functions of the hysteresis inverse model, and $q(t)$ is the inverse function of $w(t)$. In Figure 6, the ascending branch $w_{A}(t)$ and descending branch $w_{D}(t)$ are monotonic, so they are reversible. This suggests that Eq. (14) can be re-written as:

$$
\begin{aligned}
& \frac{d q_{A}}{d w}=\alpha\left[F(w)-q_{A}\right]+G(w), \quad \dot{w}>0 \\
& \frac{d q_{D}}{d w}=-\alpha\left[F(w)-q_{D}\right]+G(w), \quad \dot{w}<0
\end{aligned}
$$

where, $q_{A}(t)$ is the inverse function of $w_{A}(t)$ and $q_{D}(t)$ is the inverse function of $w_{D}(t)$. From Eq. (15) and Eq.(16), we obtain the characteristic functions:

$$
\begin{aligned}
& F(w)=\frac{1}{2}\left(q_{A}+q_{D}\right)+\frac{1}{2 \alpha}\left(\frac{d q_{A}}{d w}-\frac{d q_{D}}{d w}\right) \\
& G(w)=\frac{1}{2}\left(\frac{d q_{A}}{d w}+\frac{d q_{D}}{d w}\right)+\frac{1}{2} \alpha\left(q_{A}-q_{D}\right)
\end{aligned}
$$

Similarly, $F(w)$ and $G(w)$ can be estimated using the inverse neural network model. The transfer function $G_{2}(z)$ of the nonlinear system can be identified according to Figure 8 . It must be noted that the hysteresis inverse model must be obtained in order to negate the hysteresis non-linearities in the real system - the actuator, as shown in Figure 8.

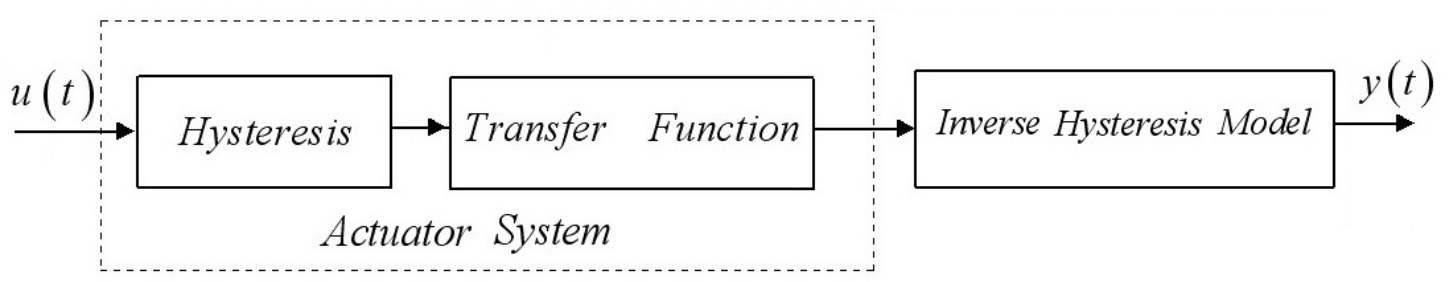

Figure 8. The block diagram of the actuator and the inverse hysteresis model to obtain a transfer function of the linear part for the actuator.

The discrete transfer function of the actuator is described by:

$$
G_{2}(z)=\frac{b_{20} z+b_{21}}{z^{2}+a_{21} z+a_{22}}
$$

As shown in Figure 5, the transfer function given by Eq.(19) is cascaded with the Duhem hysteresis model to obtain the actuator model. Figure 9 shows the results of the hysteresis system model identification errors: $e(t)$ is the error between the real actuator output $y(t)$ and the actuator system hysteresis model output $y h(t)$. The numerical values of the 
parameters in our experimentally identified model are presented in Table 2.

Table 2. The numerical values of the model parameters in Eq.19.

\begin{tabular}{|c|c|c|c|}
\hline$b_{20}$ & $b_{21}$ & $a_{21}$ & $a_{22}$ \\
\hline 0.0976 & -0.0922 & -0.6491 & -0.3453 \\
\hline
\end{tabular}

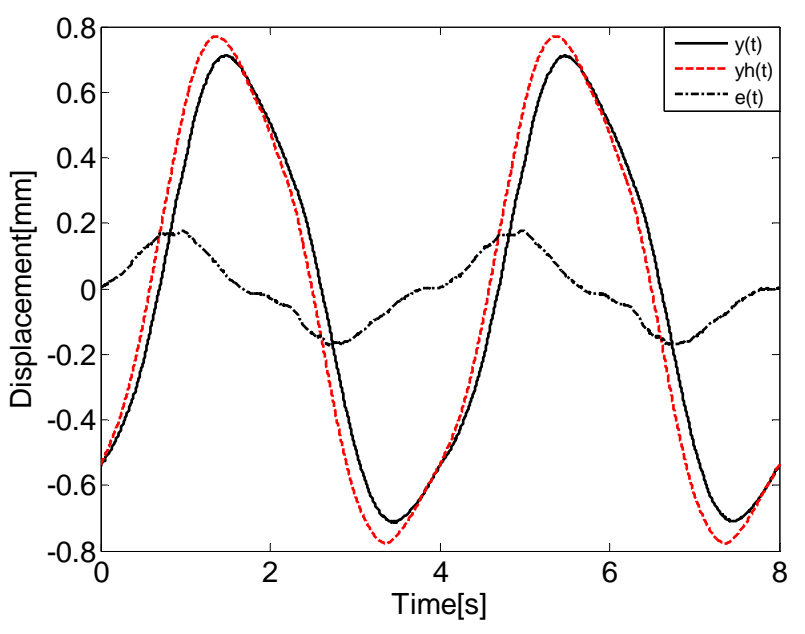

(a) Input $u(t)=\sin (0.5 \pi t)$

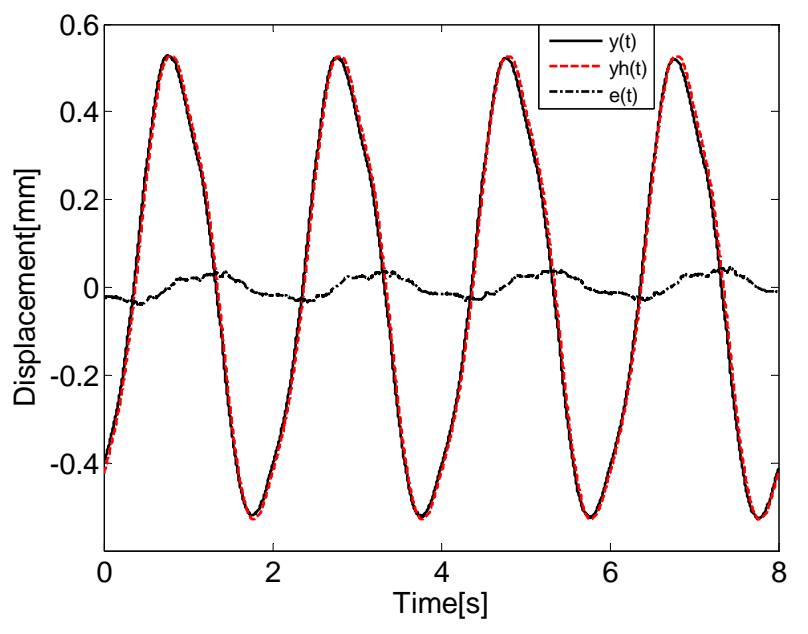

(b) Input $u(t)=\sin (\pi t)$ 


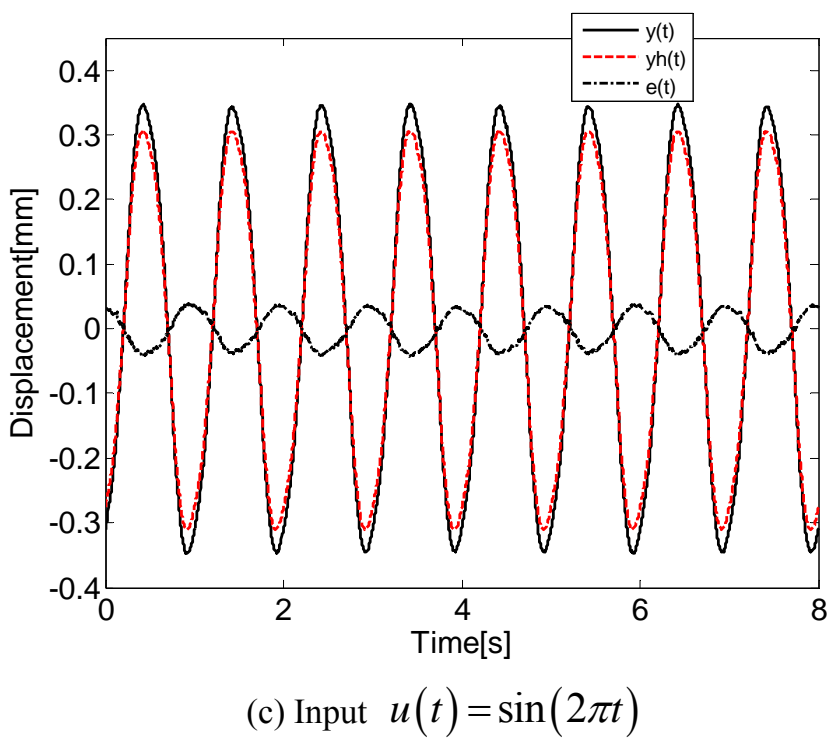

Figure 9. Comparison of the actuator + hysteresis model output and experimental actuator output under different frequencies.

To demonstrate the significance of a hysteresis model, the actuator output is calculated according to the block diagram in Figure 8, where the hysteresis model is negated by the inverse hysteresis model in order to obtain a linear transfer function. The position tracking root-mean-square (RMS) errors of these two models (with a hysteresis model and without a hysteresis model) are calculated from Eq. 20 and shown in Table 3.

$$
R M S=\sqrt{\frac{1}{m} \sum_{i=1}^{m}\left(y_{i}-y_{d i}\right)^{2}}
$$

Table 3. The RMS errors of $u(t)=\sin (\omega t)$.

\begin{tabular}{|l|c|c|c|}
\hline \multicolumn{1}{|c|}{$\omega$} & $0.5 \pi$ & $1 \pi$ & $2 \pi$ \\
\hline RMS errors of linear model & 0.1528 & 0.0371 & 0.0451 \\
\hline RMS errors of non-linear model & 0.1026 & 0.0224 & 0.0262 \\
\hline
\end{tabular}

With reference to the results in Table 3, the hysteresis model has improved the accuracy of the actuator model significantly. The RMS errors of the actuator model including hysteresis model are much smaller than those of the linear model. It follows that the proposed hysteresis modeling approach is effective. We postulate that the linear dynamics represent the electrical dynamics and the viscoelastic dynamics, while the hysteresis capture the nonlinear relationship between the stress output and the strain change induced by ion transport in and out of the PPy layers.

\section{Inversion-based feedforward control: controlling actuator output without using feedback data}

For a given conducting polymer actuator, $y_{d}(t)$ is the desired input and $y(t)$ is the real output. A desired input can be realized if the inverse actuator model compensates for the actuator dynamics and non-linearities. The two different actuator models obtained in Section 
3 will be used to develop the inverse model for the feedforward control system.

\subsection{Linear inverse model feedforward control}

Figure 10 shows the schematic of the linear inverse model $G_{1}^{-1}(z)$ feedforward controller. This model does not contain the hysteresis model. The output is given by

$$
y(t)=y_{d}(t) G_{1}^{-1}(z) G_{1}(z)
$$

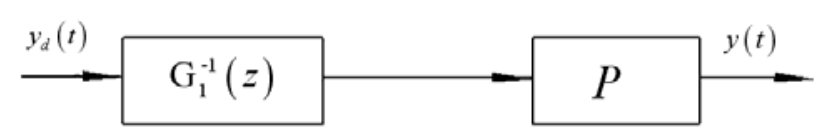

Figure 10. Schematic of the linear inverse model for the feedforward controller.

The desired output is $y_{d}(t)=\sin (\omega t)$, with a range of frequencies: $\omega(\mathrm{rad} / \mathrm{sec})=$ $0.5 \pi, 1.0 \pi, 2.0 \pi$. Based on the desired displacement, the actuator voltage signals are calculated from the inverse model. Figure 11 shows the real output $y(t)$ of the actuator, the desired output $y d(t)$ and the associated tracking errors $e(t)$.

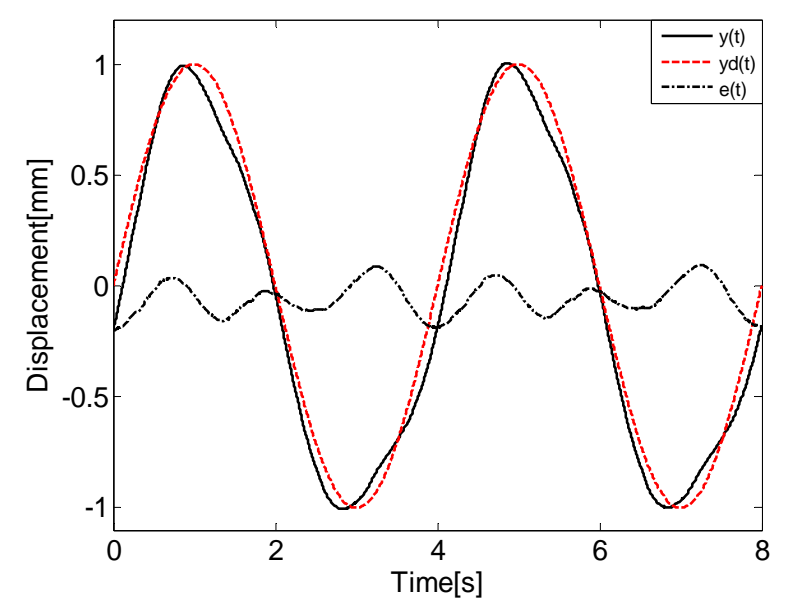

(a) Input $y_{d}(t)=\sin (0.5 \pi t)$

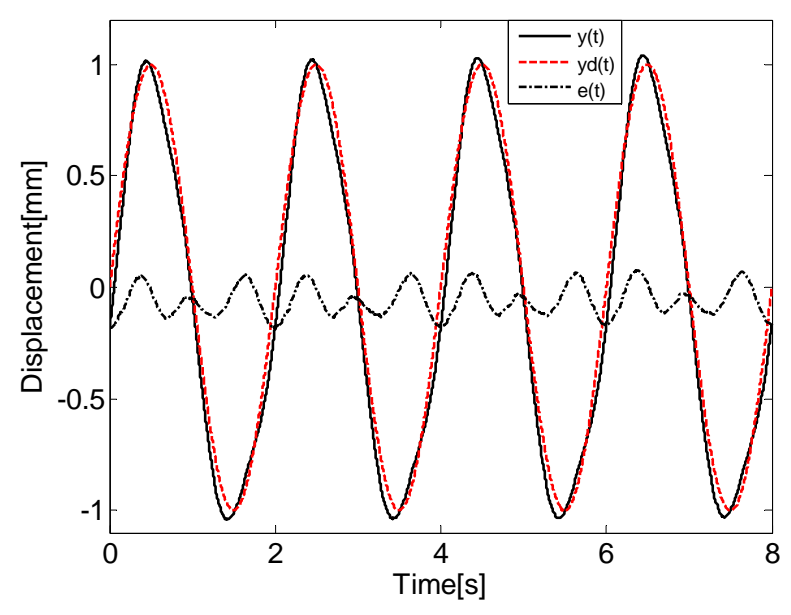

(b) Input $y_{d}(t)=\sin (\pi t)$ 


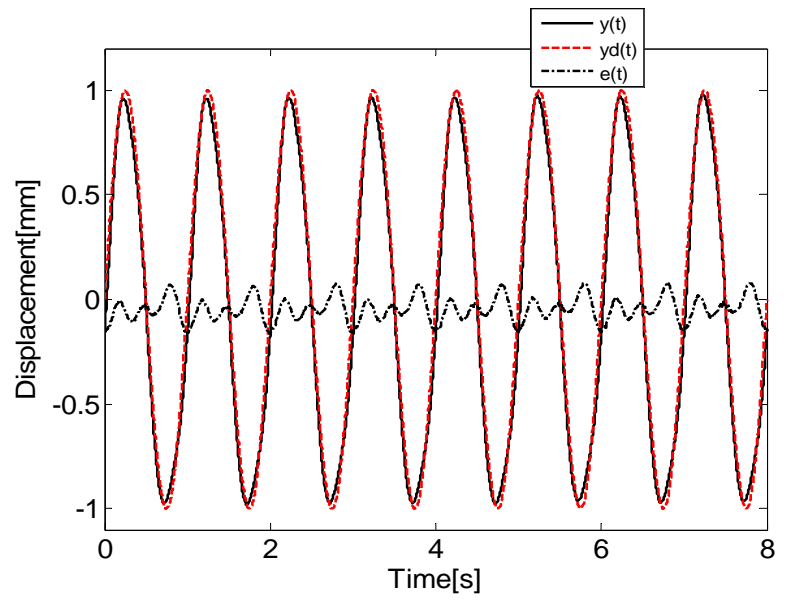

(c) Input $y_{d}(t)=\sin (2 \pi t)$

Figure 11. The real actuator output under the inverse model feedforward controller and associated tracking errors.

\subsection{Hysteresis inverse model feedforward control}

In order to control the actuator system with hysteresis, a hysteresis inverse model $H^{-1}(t)$ cascades the inverse plant model $G_{2}^{-1}(z)$ to obtain the actuator inverse model. The schematic of this inverse model feedforward controller is shown in Figure 12. The output is given by:

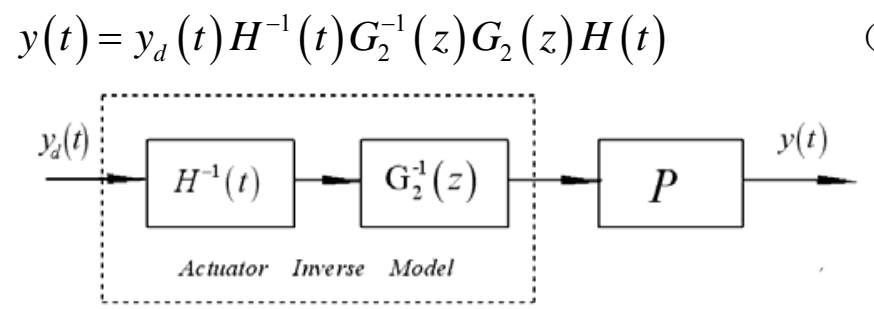

Figure 12. Schematic of the hysteresis inverse model for the feedforward controller.

The desired output is $y_{d}(t)=\sin (\omega t)$, with a range of frequencies: $\omega(\mathrm{rad} / \mathrm{sec})=$ $0.5 \pi, 1.0 \pi, 2.0 \pi$. Figure 13 shows the real output $y(t)$ of the actuator, the desired output $y d(t)$ and the associated tracking error $e(t)$. The comparison of the RMS errors of the two inverse model feedforward controllers is shown in Table 4.

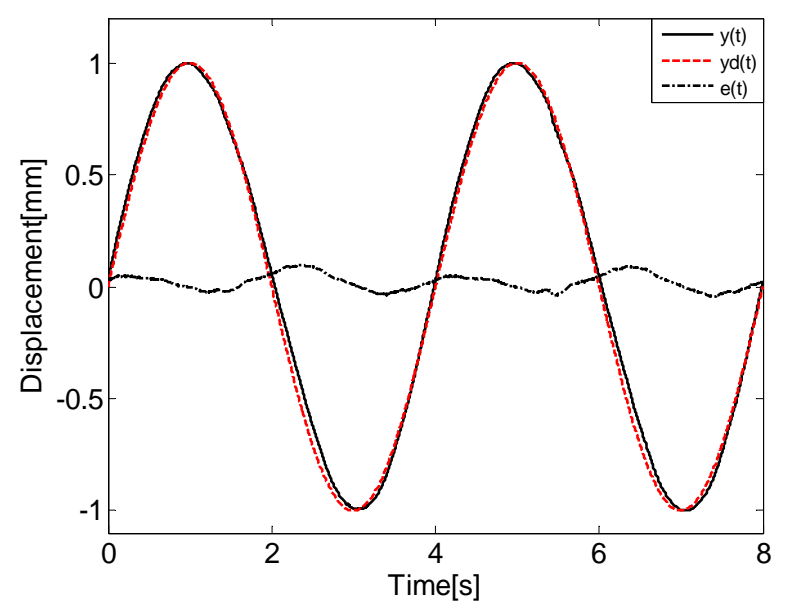


(a) Input $y_{d}(t)=\sin (0.5 \pi t)$

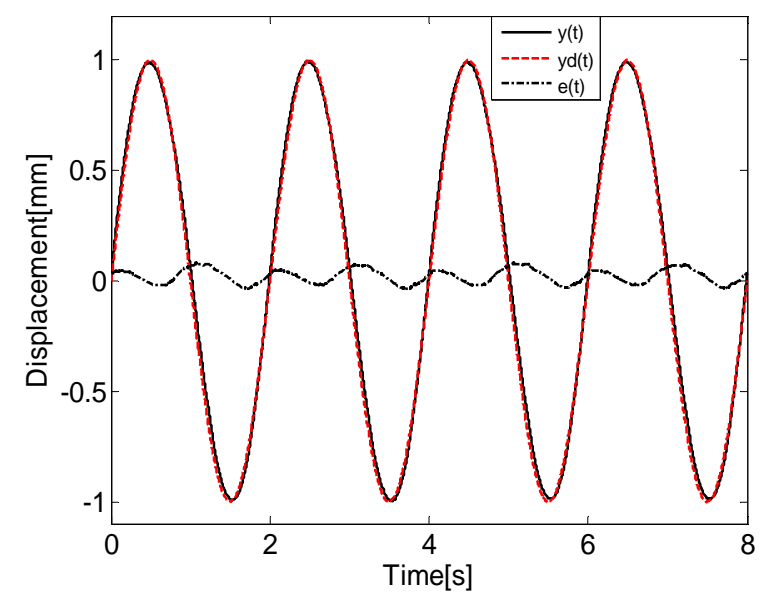

(b) Input $y_{d}(t)=\sin (\pi t)$

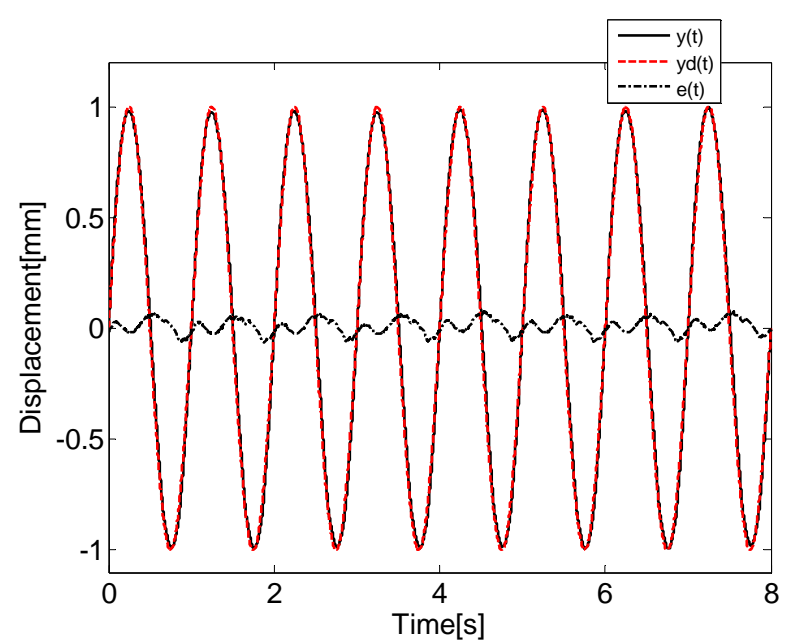

(c) Input $y_{d}(t)=\sin (2 \pi t)$

Figure 13. The real actuator output under the hysteresis inverse model feedforward controller and associated tracking errors.

Table 4. The RMS errors of $y_{d}(t)=\sin (\omega t)$.

\begin{tabular}{|l|c|c|c|}
\hline \multicolumn{1}{|c|}{$\omega$} & $0.5 \pi$ & $1 \pi$ & $2 \pi$ \\
\hline RMS errors of linear inverse model control & 0.0933 & 0.0898 & 0.0731 \\
\hline RMS errors of hysteresis inverse model control & 0.0417 & 0.0390 & 0.0344 \\
\hline
\end{tabular}

Compared with the linear inverse model control results, the performance of the inverse model control based on the proposed hysteresis model is better. The results show that the tracking errors are reduced by at least $50 \%$ if the hysteresis inverse model compensation is added to the feedforward controller. These results further demonstrate that the hysteresis modeling approach based on Duhem hysteresis model is effective for obtaining a reasonably accurate hysteresis model, and therefore an actuator model.

\section{Conclusions}


We have employed identification techniques to establish the discrete-time transfer function models for trilayer conducting polymer actuators which can both operate in dry and wet media, but the accuracy of such linear models is limited due to unmodeled non-linearities. A linear transfer function in cascade with the Duhem hysteresis model has been successfully used to generate a non-linear model of the actuator which has improved the command tracking performance of the actuators. The performance of the model including the hysteresis model was tested against the linear model. The experimental results presented show that improved performance can be obtained under different frequencies. It should be noted that after a long operation time ( $>2$ hours), the solvent evaporation can significantly change the performance of the actuator, as reported in [10]. With this in mind, the proposed inversion-based feedforward method will not function satisfactorily over extended periods of time unless the actuator is encapsulated to make sure that solvent evaporation does not occur. Otherwise, operation time-dependent dynamic models should be identified and, depending on the duration of the operation, a suitable dynamic model should be used in the feedforward controller.

Based on two identified actuator models, we implemented inversion-based feedforward control strategies and compared their tracking errors. The experimental results presented demonstrate that the hysteresis inverse model feedforward controller is the more successful control strategy. Comparing the tracking results in [24] (in particular, Figs. 18 and 19 in [24]) with those in the current work, one can see that the (open-loop) feedforward control used in this paper results in significantly lower errors. Specifically, the maximum tracking errors reported in this paper are about $5 \%$ of the amplitudes of the reference trajectories, while the maximum errors reported in [24] are $15-20 \%$ of the amplitudes of the references. This comparison shows the importance of capturing the hysteresis non-linearity in the model. The strength of the approach in [24], however, is that the (linear) model there incorporates material physics, while the linear dynamics in this work is an empirical model. A promising direction for future work, therefore, is to merge the hysteresis model presented in this work with the physics-based model in [24].

The hysteresis inverse model feedforward control strategy will help widen the implementation of smart-materials based actuators like electroactive polymer actuators in new real-world applications where external sensory feedback is impossible or costly.

\section{Acknowledgements}

This work was supported in part by the ARC Centre of Excellence for Electromaterials Science (Grant No. CE0561616) and by the US National Science Foundation (Grant No. ECCS 0547131). The first author is supported by a fellowship funded by the Chinese Scholarship Council. The authors wish to gratefully acknowledge Dr. Madeleine Strong Cincotta's help in the final language editing of this paper.

\section{References}

[1] G. Han, G. Shi, Conducting polymer electrochemical actuator made of high-strength three-layered 
composite films of polythiophene and polypyrrole, Sensors and Actuators B, 99 (2004) 525-531.

[2] B. Gaihre, G. Alici, G. M. Spinks, Julie M. Cairney, Synthesis and performance evaluation of thin film PPy-PVDF multilayer electroactive polymer actuators, Sensors and Actuators A, 165 (2011) 321-328.

[3] M. Hiraoka, P. Fiorini, J. O’Callaghan, I. Yamashita, C. Van Hoof, M. Op de Beeck, Miniature conductive polymer actuators for high pressure generation in lab onchip systems, Sensors and Actuators A, 177 (2012) 23-29.

[4] M. Fuchiwaki, K. Tanaka, K. Kaneto, Planate conducting polymer actuator based on polypyrrole and its application, Sensors and Actuators A, 150 (2009) 272-276.

[5] T. Shoa, D. S. Yoo, K. Walus, J. David, W. Madden, A Dynamic Electromechanical Model for Electrochemically Driven Conducting Polymer Actuators, IEEE/ASME Transactions on Mechatronics, 16 (2011) 42-49.

[6] G. Alici, An effective modelling approach to estimate nonlinear bending behaviour of cantilever type conducting polymer actuators, Sensors and Actuators B, 141 (2009) 284-292.

[7] P. Du, X. Lin, X. Zhang, A multilayer bending model for conducting polymer actuators, Sensors and Actuators A, 163 (2010) 240-246

[8] S. W. John, G. Alici, C. D. Cook, Inversion-Based Feedforward Control of Polypyrrole Trilayer Bender Actuators, IEEE/ASME Transactions on Mechatronics, 15(2010) 149-156

[9] Q. Yao, G. Alici, G. M. Spinks, Feedback control of tri-layer polymer actuators to improve their positioning ability and speed of response, Sensors and Actuators A. 144 (2008) 176-184.

[10] Y. Fang, X. Tan, G. Alici, Robust adaptive control of conjugated polymer actuators, IEEE Transactions on Control Systems Technology, 16 (2008) 600-612.

[11] Y. Stepanenko, C. Yi. Su, Intelligent control of piezoelectric actuators, Proceedings of the 37th IEEE Conference on Decision \& Control, (1998) 4234-4239.

[12] C. H. Ru , L. N. Sun, Hysteresis and creep compensation for piezoelectric actuator in open-loop operation, Sensors and Actuators A, 122( 2005)124-130.

[13] L. W. Chen, C. Y. Lin, C. C. Wang, Dynamic stability analysis and control of a composite beam with piezoelectric layers, Composite Structures, 56 (2002) 97-109

[14] D. Davino, C. Natale, S. Pirozzi, C. Visone, A fast compensation algorithm for real-time control of magnetostrictive actuators, Journal of Magnetism and Magnetic Materials, 290-291( 2005)1351-1354

[15] R. Iyer, X. Tan, Control of hysteretic systems through inverse compensation: Inversion algorithms, adaptation, and embedded implementation, IEEE Control Systems Magazine, 29(2009)83-99,

[16] J. Zhou, C. Wen, and Y. Zhang, Adaptive backstepping control of a class of uncertain nonlinear systems with unknown backlash-like hysteresis, IEEE Transactions on Automatic Control, 49(2004) 1751-1757.

[17] C. Y. Su, Q. Wang, X. Chen, and S. Rakheja, Adaptive variable structure control of a class of nonlinear systems with unknown prandtl-ishlinskii hysteresis, IEEE Transactions on automatic control, 50(2005)2069-2074.

[18] X. F. Zhou, S. X. Yang, G. N. Qi, X. P. Hu, Tracking control of piezoceramic actuators by using preisach model, ICMIT 2005: Control Systems and Robotics, 6042(2005) 604248.

[19] G.E. Ping, M. Jouaneh, Generalized Preisach model for hysteresis nonlinearity of piezoceramic actuators, Precision Engineering, 20 (1997) 99-111

[20] A. Dubra, J. S. Massa, Carl Paterson. Hysteresis compensation in PZT bimorph mirrors Preisachs 
classical and nonlinear models, 5th International Workshop on Adaptive Optics for Industry and Medicine, 2005,6018: 60181E

[21] X. S. Wang, H. Hong, C. Yi. Su, Adaptive Robust Control of Dynamic Systems with Unknown Input Hysteresis , The Fourth International Conference on Control and TuAO54 Automation (ICCA'03), (2003)138-142.

[22] Y. Wu, G. Alici, G.M. Spinks, G.G. Wallace, Fast trilayer polypyrrole bending actuators for high speed applications, Synthetic Metals, 156 (2006) 1017-1022.

[23] Xiangjiang Wang, Gursel Alici and Chuc Huu Nguyen, Adaptive sliding mode control of tri-layer conjugated polymer actuators, Smart Materials and Structures, 22(2013), 025004.

[24] Chuc Huu Nguyen, Gursel Alici and Gordon G. Wallace, Modelling trilayer conjugated polymer actuators for their sensorless position control, Sensors and Actuators A, 185(2012) 82-91.

[25] B. Gaihre, G. Alici, G. M. Spinks, and J. M. Cairney, "Pushing the Limits for Microactuators Based on Electroactive Polymers,” IEEE/ASME Journal of Microelectromechanical Systems, Vol.21, No.3, pp.574 585, June 2012.

[26] G. Alici, V. Devaud, P.Renaud, and G.M. Spinks, "Conducting polymer microactuators operating in air", Journal of Micromechanics and Microengineering, Vol.19, No.2, 025017, February 2009.

[27] X. S. Wang, Y. Mao, X. J. Wang, and C. Su, "Adaptive variable structure control of hysteresis in GMM actuators based on Prandtl-Ishlinskii model", $33^{\text {rd }}$ Annual Conference on the IEEE Industrial Electronics Society (IECON), pp. 721 - 726, Taipei, Taiwan, November 2007.

[28] X. Wang, G. Alici, C. Nguyen, "A Methodology to Establish a Hysteresis Model for Trilayer Conducting Polymer Actuators", 2013 IEEE/ASME International Conference on Advanced Intelligent Mechatronics, pp.111-116, Wollongong, Australia, July 2013.

[29] C. H. Nguyen, G. Alici, and G. G. Wallace, "An Advanced Mathematical Model and its Experimental Verification for Tri-layer Conjugated Polymer Actuators", IEEE/ASME Transactions on Mechatronics, August 2013, DOI: 10.1109/TMECH.2013.2280012. 